\title{
Morphological and molecular analyses of Taenia and Mesocestoides species from red foxes (Vulpes vulpes) in northwestern China
}

\section{Gang Liu}

Shihezi University

$\mathrm{Na} \mathrm{Ji}$

Shihezi University

Sándor Hornok

University of Veterinary Medicine

Yu Zhang

Shihezi University

Shanshan Zhao

Shihezi University

\section{Xueling Chen}

Shihezi University

Wurelihazi Hazihan

Shihezi University

Xinli Gu

Shihezi University

Yuan-Zhi Wang ( $\square$ wangyuanzhi621@126.com )

Shihezi University https://orcid.org/0000-0002-7500-022X

\section{Short report}

Keywords: Cestoda, tapeworms, Cyclophyllidea, Taeniidae, cox1

Posted Date: September 13th, 2021

DOl: https://doi.org/10.21203/rs.3.rs-885658/v1

License: (c) (i) This work is licensed under a Creative Commons Attribution 4.0 International License. Read Full License

Version of Record: A version of this preprint was published at International Journal for Parasitology: Parasites and Wildlife on November 1st, 2021. See the published version at 
https://doi.org/10.1016/j.ijppaw.2021.11.003. 


\section{Abstract \\ Background}

The red fox (Vulpes vulpes) is a geographically widespread predator species, occurring across the northern Hemisphere. It has high veterinary-medical significance, in part owing to its definitive host role in the life cycle of several tapeworm species, including zoonotic ones. However, there have been only few reports on cyclophyllid tapeworm (Cestoda: Cyclophyllidea) infection in red foxes from China.

\section{Methods}

In this study, 263 tapeworms were collected from eight road-killed red foxes in Xinjiang Uygur Autonomous Region (XUAR, northwestern China). The tapeworms were analyzed based on morphological characters and mitochondrial cytochrome $c$ oxidase subunit 1 (cox1) gene sequences.

\section{Results}

83 Taenia and 180 Mesocestoides tapeworms were identified according to the presence or absence of rostellum, and the number, the length and the shape of the large rostellar hooks. The morphological and molecular analyses revealed that $I$ ) 180 Mesocestoides tapeworms, here named as Mesocestoides sp. Vulpes vulpes, showed $99.21 \%$ (378/381 bp) identity to Mesocestoides sp. reported from red fox in Mongolia; and ii) 83 Taenia tapeworms belonged to three species. The first Taenia species ( $n=16$, named as Taenia sp. Vulpes vulpes), based on the length of large rostellar hooks (337-342 $\mu \mathrm{m})$ and its cox 1 sequence, was identified as a potentially novel species, which is phylogenetically close to Taenia laticollis. The second species ( $\mathrm{n}=54$, named as Taenia sp. Vulpes vulpes \& Rhombomys opimus), was morphologically similar to Taenia endothoracicus according to the number $(n=52)$, the length (319-332 $\mu \mathrm{m})$ and the shape of the large rostellar hooks. This species, infecting three red foxes, shared $100 \% \operatorname{cox} 1$ sequence identity with Taenia sp. Rhombomys opimus genotype $\mathrm{C}$ found previously in great gerbils (Rhombomys opimus) in the same region. The third species ( $n=13$, named as Taenia polyacantha-like), had shorter large rostellar hooks (178-180 $\mu \mathrm{m})$ and showed $96.27 \%(361 / 375 \mathrm{bp})$ sequence identity to Taenia polyacantha reported from red fox in Italy. In addition, co-infections with two Taenia species and with Taenia and Mesocestoides species were also demonstrated.

\section{Conclusions}

The "great gerbil-red fox" life cycle of Taenia sp. Vulpes vulpes \& Rhombomys opimus, belonging to the mitochondrial lineage of $T$. endothoracicus, is confirmed. The T. polyacantha-like species and Mesocestoides sp. Vulpes vulpes were found for the first time in red fox in China. Taenia sp. Vulpes vulpes is a potentially novel species, which is close to Taenia laticollis based on its phylogenetic 
properties. The life cycle of Taenia sp. Vulpes vulpes and Mesocestoides sp. Vulpes vulpes should be further explored.

\section{Background}

The red fox (Vulpes vulpes Linnaeus, 1758), that is the largest species of true foxes (Carnivora: Canidae: Vulpes), is widespread in North-America, Asia, Europe and North Africa [1]. Its habitats overlap with activity areas of humans and domestic animals, leading to risks of infectious diseases (e.g. rabies and echinococcosis) relevant to public and veterinary health $[2,3]$.

Tapeworms (cestodes) have a domestic or sylvatic life cycle based on predator-prey interactions between a carnivore (definitive host) and a herbivore (intermediate host) [4]. Previously, the red fox was reported to harbor at least 22 species of cyclophyllidean cestodes, including Taenia taeniaeformis, Taenia pisiformis, Taenia polyacantha, Taenia hydatigena, Taenia multiceps, Taenia pisiformis, Taenia serialis, Taenia crassiceps, Taenia ovis, Taenia polyacantha, Taenia spp., Echinococcus multilocularis, Echinococcus perfoliatus, Echinococcus granulosus, Echinococcus shiquicus, Mesocestoides lineatus, Mesocestoides corti, Mesocestoides spp., Dipylidium caninum, Diplopylidium noelleri, Alaria alata, and Hymenolepis spp. [5-25].

Previously, Echinococcus granulosus, E. multilocularis, and E. shiquicus were found in Tibetan fox (Vulpes ferrilata) and red fox in China [25]. The aim of the present study was to identify tapeworms from road-killed red foxes in Xinjiang Uygur Autonomous Region (XUAR), northwestern China.

\section{Methods}

\section{Sample collection}

In this study, eight red foxes were used. These animals were road-killed in the Gurbantunggut Desert, the northern region of XUAR in the period 2015-2019. During necropsy, all tapeworms were removed from the small intestine (Table S1, Fig. S1), subsequently washed in physiological saline prior to morphological identification and DNA extraction.

\section{Morphological identification}

The staining procedure was performed as reported [26]. In brief, the scolex of representative tapeworms were stained with acetate carmine and observed with an Olympus DP70 digital camera (Olympus, Tokyo, Japan).

\section{DNA extraction and molecular analysis}

After detailed morphological examination, 40 tapeworm specimens were selected for molecular analysis (as shown in Table S1). DNA was extracted from a small part of the immature strobila using the TIANamp Genomic DNA Kit (TIANGEN, Beijing, China). A conventional PCR was performed to amplify an 
approx. 450-bp-long part of the mitochondrial cytochrome coxidase subunit I gene (cox1) as reported previously [27]. Sequences from this study were compared to those in GenBank with the BLASTn program (https://blast.ncbi.nlm.nih.gov). New sequences were deposited in GenBank (cox1: MZ475884, MZ512998-MZ513000). A phylogenic tree was constructed using the Neighbor-Joining method in MEGA 7.0 software.

\section{Results}

\section{Morphological identification}

Six foxes (\#1, \#3, \#5, \#6, \#7 and \#8) were found infected with 263 tapeworms. According to the presence or absence of rostellum, 83 were identified as Taenia spp., while 180 belonged to the genus Mesocestoides. According to the number, the length and measurement data (total length, total width, posterior length, anterior length and guard length) of large rostellar hooks, 83 Taenia tapeworms were assigned to three species. The first species ( $n=16$, removed from red fox \#1) and the second species ( $n=54$, from red foxes \#3, \#5, \#7 and \#8) were similar to Taenia endothoracicus, Taenia krepkogorski and Taenia macrocystis in the number and length of large rostellar hooks (Table S2, Table S3). The third species ( $n=13$, from red foxes \#5, \#6 and \#8) belonged to Taenia polyacantha according to the number and length of large rostellar hooks.

\section{Molecular identification}

Based on the $\operatorname{cox} 1$ gene, BLASTN analysis indicated that i) all 180 Mesocestoides tapeworms from red foxes \#1 $(n=80)$ and \#3 $(n=100)$ were most closely related to Mesocestoides sp. (accession number: AB792718) from red fox in Mongolia, showing $99.21 \%$ (378/381 bp) sequence identity to it. Provisionally, this species was named as Mesocestoides sp. Vulpes vulpes (MZ475884); ii) the first Taenia species was most closely related to Taenia sp. Rhombomys opimus (accession numbers: MW321849 and MW321850) detected in great gerbils in our previous work [28], but sharing only $88.03 \%$ (309/351 bp) sequence identity with it. Provisionally, this species was named as Taenia sp. Vulpes vulpes (MZ512998), and it belonged to Clade B of the cox 1 phylogenetic tree (Fig. 1); iii) the second Taenia species was $100 \%$ identical in its cox 1 sequence to Taenia sp. Rhombomys opimus genotype C (accession numbers: MW321849 and MW321850) detected in great gerbils (Rhombomys opimus), originating from the same region as the relevant three road-killed red foxes (\#5, \#7 and \#8) in this study. Provisionally, this species was named as Taenia sp. Vulpes vulpes \& Rhombomys opimus (MZ512999), and belonged to Clade A of the cox1 phylogenetic tree (Fig. 1); and iv) the third Taenia species was most closely related to Taenia polyacantha (accession number: MT806362) from red fox in Italy, sharing $96.27 \%$ (361/375 bp) cox 1 sequence identity. Provisionally, this species was named as "Taenia polyacantha-like" (MZ513000), and it belonged to Clade C of the cox1 phylogenetic tree (Fig. 1).

Analysis of COX1 amino acid sequences showed that i) Mesocestoides sp. Vulpes vulpes has 100\% (127/127 aa) identity to Mesocestoides sp. (accession number: AB792718) from red fox in Mongolia; ii) 
Taenia sp. Vulpes vulpes shares $93.16 \%$ (109/117 aa) identity with Taenia sp. Rhombomys opimus (MW321849) (Fig. S2A); and iii) "T. polyacantha-like" (from Clade C in Fig. 1) has $100.00 \%$ (125/125 aa) identity to Taenia polyacantha (MT806362) (Fig. S2B).

\section{Discussion}

The previously known geographical range of Taenia endothoracicus, with red fox as its definitive host, included Iran and North Africa. Polycephalic larvae occur in the thoracic cavity of rodents from the genera Meriones, Gerbillus and Rhombomys. In our previous work, Taenia sp. Rhombomys opimus genotypes A, $B$ and $C$ were demonstrated from the thoracic cavity of great gerbils in Gurbantungut Desert with $5.3 \%$ (10/188) prevalence [28]. A lot of protoscolices were observed in large white and web-shaped cysts, which caused shrinking and stiffness in the lung parenchyma [28]. Interestingly, here adults of Taenia sp. Vulpes vulpes \& Rhombomys opimus were found in four red foxes, and the corresponding cox 1 sequence had $100 \%$ identity to that of Taenia sp. Rhombomys opimus genotype $\mathrm{C}$ in great gerbils. Furthermore, the sampling sites of the three red foxes (\#5, \#7 and \#8) and of the two great gerbils (\#27 and \#31) infected with Taenia sp. Rhombomys opimus genotype $\mathrm{C}$ are situated in the same area (44. $67^{\prime}-44^{\circ} 70^{\prime} \mathrm{N}$, $86^{\circ} 34^{\prime}-86^{\circ} 39^{\prime}$ E) [28]. Previously, Lin et al reported that great gerbils are important in the diet composition of red foxes in Gurbantungut Desert, and accounted for $16.48 \%$ of prey items based on 273 fecal samples of red foxes [29]. These findings suggest that the life cycle of Taenia sp. Vulpes vulpes \& Rhombomys opimus involves the great gerbil and the red fox (Fig. S3). In addition, the shape of large rostellar hooks was compared among Taenia sp. Vulpes vulpes \& Rhombomys opimus, Taenia endothoracicus, Taenia krepkogorski and Taenia macrocystis (as shown in S pptx). The results indicated that the shape of large rostellar hooks is similar between T. endothoracicus and Taenia sp. Vulpes vulpes \& Rhombomys opimus. Interestingly, the shape of cysts showed significant difference between $T$. endothoracicus reported from Iran and Taenia sp. Rhombomys opimus in this work (Fig. S4) [28, 30, 31], suggesting that Taenia sp. Vulpes vulpes \& Rhombomys opimus belongs to a genetic lineage separate from $T$. endothoracicus. However, unfortunately, the cox 1 sequence of the latter species is not available in GenBank to confirms this.

According to the length of the large rostellar hooks and definitive host (Vulpes), Taenia sp. Vulpes vulpes is similar to Taenia endothoracicus, Taenia krepkogorski and Taenia macrocystis. However, when the shape of the large rostellar hooks was compared among them, Taenia sp. Vulpes vulpes showed obvious differences from these three tapeworm species (as shown in S pptx). Due to the lack of cox 1 data of $T$. endothoracicus, T. krepkogorski and T. macrocystis, the taxonomy of Taenia sp. Vulpes vulpes should be further investigated in the future.

Tania polyacantha, infecting red foxes, was previously reported from Finland, Norway, Denmark, Wales, Germany, France, Switzerland, Austria, Poland, Bulgaria, Italy, Yugoslavia and Japan [32, 33]. In this study, 13 adult tapeworms of "Taenia polyacantha-like" were found for the first time in three red foxes (\#5, \#6 and \#8) in China. At the same time, differences between these isolates are obvious in the shape of the large rosteller hooks and sequences of the cox1 gene (96.27\%, 361/375 bp). 
Previously, M. lineatus, M. corti and Mesocestoides spp. were found in red foxes in Portugal, Romania, Denmark, Italy and Mongolia [6, 7, 13, 17]. Hitherto only $M$. lineatus was reported from this genus in China [34]. In our study, however, Mesocestoides sp. Vulpes vulpes (accession number: MZ475884) was found in two red foxes. This species candidate is phylogenetically most closely related to Mesocestoides sp. (accession number: AB792718) collected from red fox (Vulpes vulpes) in Mongolia, with which it shared 99.21\% (378/381 bp) cox1 sequence homology. These two clustered as a sister group to $M$. corti and Mesocestoides canislagopodis (Fig. 1). Since the latter species has a broad range of canid and felid definitive hosts [35], it should be examined in the future if further predator species may harbor adult Mesocestoides sp. Vulpes vulpes in northwestern China. In addition, the taxonomic relationships and the geographical distribution of Mesocestoides sp. Vulpes vulpes should also be explored.

It is a principle phenomenon that coinfection with multiple tapeworm species may occur in the same red fox individual. In a previous report from the Netherlands, up to eight cestode species were found together in the same red fox [11]. Here Taenia sp. Vulpes vulpes coinfected with Mesocestoides sp. Vulpes vulpes, "T. polyacantha-like" coinfected with Taenia sp. Vulpes vulpes \& Rhombomys opimus the same red fox in China. The broad range of food items of red foxes in the Gurbantunggut Desert might account for this coinfection phenomenon.

\section{Conclusion}

The life cycle of Taenia sp. Vulpes vulpes \& Rhombomys opimus, belonging to the mitochondrial lineage of $T$. endothoracicus, involves the great gerbil and the red fox. "Taenia polyacantha-like" and Mesocestoides sp. Vulpes vulpes were found for the first time in red fox in China. Taenia sp. Vulpes vulpes, a potentially novel species, is phylogenetically closely related to $T$. laticollis based on the cox 1 gene.

\section{Abbreviations}

cox1: cytochrome coxidase subunit I; XUAR: Xinjiang Uygur Autonomous Region.

\section{Declarations}

\section{Acknowledgements}

The authors thank the contributions by the staff at the School of Medicine and School of Animal Science and Technology, Shihezi University.

\section{Authors' contributions}

GL, YW conceived and designed the study, and wrote the manuscript. GL, NJ, YZ, SZ, XC, WH, XG and YW performed the experiments, analyzed the data. SH contributed to study design and edited the manuscript. All authors read and approved the final manuscript. 


\section{Funding}

This work was supported by the National Natural Science Foundation of China (81960379 and 31960709), International Scientific and Technological Cooperation in Bingtuan (2020BC008) and Nonprofit Central Research Institute Fund of Chinese Academy of Medical Sciences (2020-PT330-003).

\section{Availability of data and materials}

The sequences obtained and analysed during the present study are deposited in the GenBank database, under the accession numbers (tapeworms cox1 gene: MZ475884, MZ512998, MZ512999 and MZ513000 ) .

\section{Ethical approval and consent to participate}

This study was approved by the Animal Ethics Committee of Shihezi University (Approval No. A2015-06301 and A2018-143-01).

\section{Consent for publication}

Not applicable.

\section{Competing interests}

The authors declare that they have no competing interests.

\section{Author details}

${ }^{1}$ Department of Basic Medicine, School of Medicine, Shihezi University, Shihezi, Xinjiang Uygur Autonomous Region, China. ${ }^{2}$ Department of Parasitology and Zoology, University of Veterinary Medicine, Budapest, Hungary. ${ }^{3}$ Department of Veterinary Medicine, College of Animal \& Science, Shihezi University, Shihezi, Xinjiang Uygur Autonomous Region, China. ${ }^{4} \mathrm{NHC}$ Key Laboratory of Prevention and Treatment of Central Asia High Incidence Diseases, First Affiliated Hospital, School of Medicine, Shihezi University, Shihezi City, Xinjiang Uygur Autonomous Region, China.

\section{References}

1. Ablimiti AQ. Classification and distribution of mammals in Xinjiang[M]. Beijing: Science Press; 2013.

2. Centers for Disease Control and Prevention (CDC). Human rabies-Texas and New Jersey, 1997. MMWR Morb Mortal Wkly Rep. 1998;47:1-5.

3. Wang Z, Wang X, Liu X. Echinococcosis in China, a review of the epidemiology of Echinococcus spp. Ecohealth. 2008;5:115-26.

4. Thompson RCA. Parasite zoonoses and wildlife: one health, spillover and human activity. Int $J$ Parasitol. 2013;43:1079-88. 
5. Abuladze KI. "Principles of Cestodology, edited by K. I. Skryabin. Vol. IV. Taeniata-cestodes of animals and man and the diseases caused by them." (1964).

6. Eira C, Vingada J, Torres J, Miquel J. The Helminth Community of the Red Fox, Vulpes Vulpes, In Dunas de Mira (Portugal) and its effect on host condition. Wildlife Biology in Practice. 2006;2:26-6.

7. Barabási SS, Fok E, Gubányi A, Mészáros F, Cozma V. Helminth fauna of the small intestine in the European red fox, Vulpes vulpes with notes on the morphological identification of Echinococcus multilocularis. Sci Parasitol. 2010;11:141-51.

8. Hofer S, Gloor S, Muller U, Mathis A, Hegglin D, Deplazes P. High prevalence of Echinococcus multilocularis in urban red foxes (Vulpes vulpes) and voles (Arvicola terrestris) in the city of Zürich, Switzerland. Parasitology. 2000;120:135-42.

9. Laurimaa L, Moks E, Soe E, Valdmann H, Saarma U. Echinococcus multilocularis and other zoonotic parasites in red foxes in Estonia. Parasitology. 2016;143:1450-8.

10. Dybing NA, Fleming PA, Adams PJ. Environmental conditions predict helminth prevalence in red foxes in Western Australia. Int J Parasitol Parasites Wildl. 2013;2:165-72.

11. Franssen F, Nijsse R, Mulder J, Cremers H, Dam C, Takumi K, et al. Increase in number of helminth species from Dutch red foxes over a 35-year period. Parasit Vectors. 2014;7:166.

12. Karamon J, Dąbrowska J, Kochanowski M, Samorek-Pieróg M, Sroka J, Różycki M, et al. Prevalence of intestinal helminths of red foxes (Vulpes vulpes) in central Europe (Poland): a significant zoonotic threat. Parasit Vectors. 2018;11:436.

13. Saeed I, Maddox-Hyttel C, Monrad J, Kapel CM. Helminths of red foxes (Vulpes vulpes) in Denmark. Vet Parasitol. 2006;139:168-79.

14. Borgsteede FHM. Helminth parasites of wild foxes (Vulpes vulpes $L$.) in The Netherlands. $Z$ Parasitenkd. 1984;70:281-5.

15. Morishima Y, Tsukada H, Nonaka N, Oku Y, Kamiya M. Coproantigen survey for Echinococcus multilocularis prevalence of red foxes in Hokkaido, Japan. Parasitol Int. 1999;48:121-34.

16. Petersen HH, Al-Sabi MNS, Enemark HL, Kapel CMO, Jørgensen JA, Chriél M. Echinococcus multilocularis in Denmark 2012-2015: high local prevalence in red foxes. Parasitol Res. 2018;117:2577-84.

17. Fontana M. C, et al. Helminth parasites of the red fox Vulpes vulpes (L. 1758) and the wolf Canis Iupus italicus Altobello, 1921 in Emilia-Romagna, Italy. Ital J Zool. 2016;83:503-13.

18. Brochier B, De Blander H, Hanosset R, Berkvens D, Losson B, Saegerman C. Echinococcus multilocularis and Toxocara canis in urban red foxes (Vulpes vulpes) in Brussels, Belgium. Prev Vet Med. 2007;80:65-73.

19. Hanosset R, Saegerman C, Adant S, Massart L, Losson B. Echinococcus multilocularis in Belgium: prevalence in red foxes (Vulpes vulpes) and in different species of potential intermediate hosts. Vet Parasitol. 2008;151:212-7. 
20. Rataj AV, Bidovec A, Ele D, Vengut G. Echinococcus multilocularis in the red fox (Vulpes vulpes) in Slovenia. Eur J Wildlife Res. 2010;56:819-22.

21. Romig T, Thoma D, Weible AK. Echinococcus multilocularis - A zoonosis of anthropogenic environments? J Helminthol. 2006;80:207-12.

22. Mrcvs IWBBM. Echinococcus multilocularis: a political zoonosis. Companion Animal. 2013;18:36871.

23. Dibble ED, Font WF, Wittrock DD. Helminths of the red fox, Vulpes vulpes $L$. in west central Wisconsin. J Parasitol. 1983;69:1170-2.

24. Loos-Frank B. Shedding of gravid proglottids and destrobilation in experimental infections of foxes with Mesocestoides leptothylacus Loos-Frank, 1980 (Cestoda). J Helminthol. 1987;61:213-8.

25. Jiang W, Liu N, Zhang G, Renqing P, Xie F, Li T, et al. Specific detection of Echinococcus spp. from the Tibetan fox (Vulpes ferrilata) and the red fox ( $V$. vulpes) using copro-DNA PCR analysis. Parasitol Res. 2012;111:1531-9.

26. Li XJ, Yang YM. Identification of Taenia cestodes in Xianggelila, Yunnan by molecular and morphological methods. J Pathogen Biol. 2009;4:196-7.

27. Hrčkova G, Miterpáková M, O'Connor A, Šnábel V, Olson PD. Molecular and morphological circumscription of Mesocestoides tapeworms from red foxes (Vulpes vulpes) in central Europe. Parasitology. 2011;138:638-47.

28. Ji N, Chen X, Liu G, Zhao S, Tan W, Liu G, et al. Theileria, Hepatozoon and Taenia infection in great gerbils (Rhombomys opimus) in northwestern China. Int J Parasitol Parasites Wildl. 2021;15:79-86.

29. Lin X, Wu K, Shi L. Food habitats of the red fox (Vulpes vulpes) in the Junggar Basin Desert. Acta Theriologica Sinica. 2010;30:346-50.

30. Mowlavi GR, Kia EB, Mobedi I. Natural infection of the gerbil Meriones lybicus with the metacestode of Taenia endothoracicus in Arak, central Iran. J Helminthol. 2004;78:275-6.

31. Kamranrashani B, Kia E, Mobedi I, Mohebali M, Zarei Z, Mowlavi G, et al. Helminth Parasites of Rhombomys opimus from Golestan Province, Northeast Iran. Iran J Parasitol. 2013;8:78-84.

32. Loos-Frank B, Zeyhle E. The intestinal helminths of the red fox and some other carnivores in southwest Germany. Z Parasitenkd. 1982;67:99-113.

33. Ihama Y, Sato H, Makino Y, Kamiya H. Two Taenia species found in Japan, with new distribution record of Taenia polyacantha Leuckart, 1856 (Cestoda: Taeniidae). Parasitol Int. 2000;48:303-6.

34. Zhou XN. list of parasitic germplasm resources in China (cestode)[M]. Shanghai China: Shanghai Science and Technology Press; 2015.

35. Skirnisson K, Jouet D, Ferté H, Nielsen ÓK. Occurrence of Mesocestoides canislagopodis (Rudolphi, 1810) (Krabbe, 1865) in mammals and birds in Iceland and its molecular discrimination within the Mesocestoides species complex. Parasitol Res. 2016;115:2597-607.

\section{Figures}




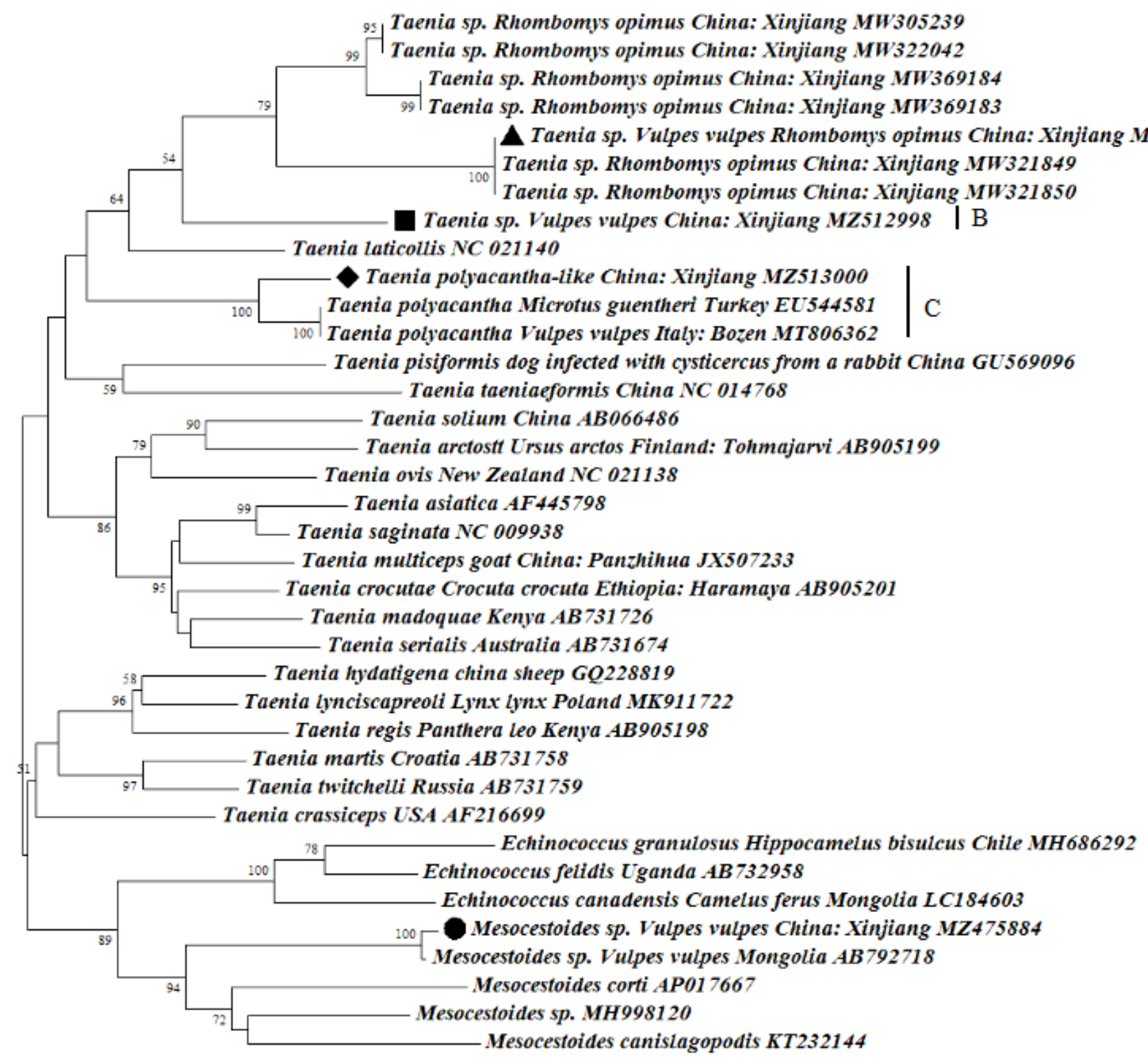

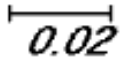

Figure 1

Phylogenetic relationships of Taenia and Mesocestoides species from red foxes based on cox 1 sequences.

\section{Supplementary Files}

This is a list of supplementary files associated with this preprint. Click to download.

- GraphicalAbstract.png

- SupplePPTX.pptx

- SupplementaryFig.1.tif 
- SupplementaryFig.2.tif

- SupplementaryFig.3.tif

- SupplementaryFig.4.tif

- SupplementaryTable1.doc

- SupplementaryTable2.doc

- SupplementaryTable3.doc 